\title{
Traducción al español de una selección de poemas de Mary O'Donnell
}

\author{
Inés Praga-Terente
}

Universidad de Burgos, Spain

Copyright (c) 2014 by Inés Praga-Terente. This text may be archived and redistributed both in electronic form and in hard copy, provided that the author and journal are properly cited and no fee is charged for access.

La traducción de estos siete poemas de Mary O'Donnell (en una selección realizada por la propia autora) ha supuesto un breve pero intenso viaje a través de su universo poético. Novelista y autora de relatos cortos, además de ensayista y traductora ${ }^{1}$ Mary O'Donnell se declara sobre todo poeta

cuando vienen los poemas vivo con ellos y a través de ellos. Es la parte más espiritual de mi vida. Normalmente los poemas surgen a lo largo de un periodo de meses. Y luego hay un silencio, un período de barbecho que necesito para recuperarme. ... Un poema es una esencia intensa, como un pinchazo que te llena de alegría, a medio camino entre el dolor y el placer. $^{2}$

Tal esencia intensa preside sin duda esta selección de poemas, un arpegio que alterna el dolor y el placer y que recorre con maestría vivencias y formas variadas y complejas. Por ello

1. Mary O’Donnell es autora de once libros. De ellos destacaremos sus aclamadas novelas The Light-Makers (1992) y The Elysium Testament (1999) y en poesía Unlegendary Heroes (1998), The Place of Miracles. New and Selected Poems (2006) y The Ark Builders (2009). Asimismo es la coeditora de una antología de poetas irlandesas traducidas al inglés y al irlandés: To the Winds our Sails. Irish Writers Translate Galician Poetry (2010). En el verano de 2014 proyecta la publicación de un nuevo poemario, The Parts.

2. González Arias, Luz Mar, "Poemas desde las fronteras del arte: conversaciones con Mary O'Donnell y Celia de Fréine" en Palacios, Manuela y González Fernández, Helena (eds). Palabras Extremas: Escritoras gallegas e irlandesas de hoy. A Coruña: Netbiblo, 2008: 149. La traducción de estas palabras es de Luz Mar González Arias. pregunta: ¿es posible traducir la intensidad, la esencia, la emoción, el alma de las palabras y las cosas, esos elementos intangibles que confieren a la poesía su modo de ser? La propia autora, traductora y persona muy implicada en esta disciplina formula los mismos interrogantes:

It remains one of the essential questions whenever translation is in the air: how should it be done - an attempt at a literal transposition, an attempt to capture the spirit of the poem, regardless what gymnastics of language and phrasing, or is it a bit like making a dog stand on its hind legs. In other words, can it be done at all?

Y responde a ello dejando en manos del traductor la elección que dependería de "a matter of discernment, personal approach and sometimes political strategy - conscious and unconscious on the translator's part". ${ }^{3}$ También subraya un elemento importante, que es la identidad cultural del autor/a de la obra y la necesaria familiaridad y comprensión de la misma por parte del traductor.

3. O'Donnell, Mary: "Introduction" en O'Donnell, Mary y Palacios, Manuela (eds.), To the Winds our Sails. Irish Writers Translate Galician Poetry. Salmon Poetry, 2010: 19. Trad. "Es una de las pregunta esenciales cuando hay que resolver una traducción: ¿Cómo hay que hacerla? ¿Intentando una transposición literal o intentando capturar el espíritu del poema, sin tener en cuenta toda clase de gimnasia lingüística o de estilo? ¿O es un poco como hacer a un perro sostenerse en sus patas traseras? En otras palabras, ¿Realmente se puede hacer?; "es una cuestión de criterio, enfoque personal y estrategia política -consciente o inconsciente - por parte del traductor." 
En esta introducción se pretende aclarar muy brevemente nuestro posicionamiento a la hora de abordar estos poemas. Por un lado, la familiaridad y el entendimiento de la identidad cultural de la autora no han supuesto mayor problema, por cuanto se engarza en una/s tradición/es y un contexto sobre los que he trabajado ampliamente. Bien es cierto que su nacimiento y crianza en el fronterizo condado de Monaghan - entre Irlanda del Norte y la República - la hace partícipe y conocedora de dos mundos cercanos y a la vez muy distantes, algo que ya sucedió a otros grandes poetas como Patrick Kavanagh y Seamus Heaney. En cuanto a nuestra fe y nuestras estrategias en la traducción, invocamos y compartimos un texto de Octavio Paz - gran traductor él mismo - que rechaza la idea de que la poesía sea intraducible a pesar de definirla como ese "tejido de connotaciones hecho de ecos, reflejos y correspondencias entre el sonido y el sentido". Asimismo compartimos su enfoque, a medio camino entre la pura literalidad y la traducción libre del traduttore/tradittore:

Los sentidos de un poema son múltiples y cambiantes mientras que sus palabras son únicas e insustituibles. Cambiarlas sería destruir el poema. Pero la poesía, sin cesar de ser lenguaje, es un más allá del lenguaje. El punto de partida del traductor no es el lenguaje en movimiento, materia prima del poeta, sino el lenguaje fijo del poema. Lenguaje congelado y no obstante perfectamente vivo. $\mathrm{Su}$ operación es inversa a la del poeta: no se trata de construir con signos móviles un texto inamovible sino de desmontar los elementos de ese texto, poner de nuevo en circulación los signos y devolverlos al lenguaje. El ideal de la traducción poética, consiste en producir con medios diferentes efectos análogos. ${ }^{4}$

Es curioso que uno de los poemas de este corpus, "Hungary", aborde directamente la labor traductora y que en un juego de palabras imposible de traducir - el doble sentido de "translate" como "trasladar" y "traducir" - la compare al movimiento de las nubes "trasladándose/traduciendo de un país a otro". Porque para Mary O'Donnell la traducción crea puentes y forja un canal de doble dirección entre dos culturas, siendo un instrumento precioso para reconocer y acercar lo que es igual y/o diverso, para

4. Paz, Octavio. Traducción: literatura y literalidad. Barcelona: Tusquets Editores, 1971: 15-16. favorecer el intercambio y el dialogo. ${ }^{5}$ Por otra parte, las palabras de Octavio Paz hablan de poner en circulación un lenguaje fijo pero vivo, en definitiva de reactivar y reanimar un poema por medio de la traducción sin traicionar su esencia. Todo ello implica necesariamente algunos pasos pero sobre todo la acción de "desmontar los elementos de un texto", entendiendo por ello el análisis riguroso de la individualidad de cada poema y de las claves que le confieren su modo de ser. De ahí se deriva el reconocimiento (y la reproducción en otra lengua) de la amplia gama de registros que O'Donnell utiliza, esa pluralidad de vivencias y códigos que encierran los poemas seleccionados y que les otorga una gran riqueza.

El primero de ellos, "Feeding the Crone", recrea el antiquísimo mito de la personificación de Irlanda como una anciana achacosa que ha adoptado diversos nombres - Shan Van Vocht, Cailleach, Old Woman of Beara - y que se ha propagado como emblema de longevidad e inmortalidad. Esta es la clave con que debemos abordar el poema, un poema rico en ironía al igual que el pastel que alimenta a la vieja bruja es "rico en juventud". La noción de fertilidad nutre sus estrofas con un tono coloquial y un léxico cotidiano que (de)construye uno de los mitos más poderosos del imaginario irlandés. Por otra parte, no es infrecuente en O'Donnell la referencia al cuerpo de la mujer en su imperfección - ya sea por ancianidad $\mathrm{u}$ otros aspectos $-\mathrm{y}$ a las mujeres de espíritu incontrolable e indómito como la vieja del poema.

La maternidad - otro elemento recurrente en su poética - es el centro de "The Cosmos Ticked Silently", un poema de muy distinto signo y en cierto modo complementario de la visión de feminidad/fertilidad que nos ofrecía el anterior. Nótese, por ejemplo, que en los dos hay referencias al vientre de la mujer pero con un sentido muy diferente. Aquí se aborda uno de los iconos religiosos femeninos más importantes para la autora, la Virgen María, que representa un alivio para el crudo destino de la Sagrada Familia con el bálsamo de su amor y ternura de madre. Los largos versos del poema y los frecuentes encabalgamientos parecen querer abarcar la infinitud de un cosmos que avanza majestuoso y que contrasta con la entrañable pero mísera

5. González Arias, Luz Mar . Ob. Cit. 2010: 147. 
imagen de la familia en su intimidad. Puede afirmarse que la vida, el nacimiento, la fertilidad, en definitiva los mitos genésicos son muy queridos para la autora como a su vez lo son los mitos apocalípticos. No es extraño, por tanto, que el poema siguiente "Spring Funeral", sea una (des) dramatización de la muerte en solemnes pareados y cierto tono tragicómico que hacen avanzar por la nave de una representación cuasi-teatral a sus histriónicos personajes. Estos dos poemas, muy distintos en letra y espíritu, junto con "Feeding the Crone", prueban el arraigo de la autora en la mitología y las referencias culturales y a la vez su actitud iconoclasta hacia ellas.

El quinto poema, "Eden" nos muestra una estrategia muy distinta como es la evocación, en este caso del universo de la infancia y del magnetismo del mundo natural, elemento clave para O'Donnell. Los ecos del huerto de su infancia se plasman en una poesía sensorial, sonora, llena de olores y sabores, de sensaciones mágicas y al mismo tiempo épicas. La magia se prolonga a su vez en la contemplación que vertebra los dos poemas finales, "Buzzard" y "Pleasure", dos instantes de emoción y asombro ante la belleza del mundo animal y de la naturaleza circundante. En este caso el registro léxico es minucioso, denso, una auténtica filigrana que parece dibujar las aves y desgranar sus movimientos majestuosos o frágiles -, un tapiz de palabras de notable complejidad para la traducción. Porque Mary O'Donnell es a veces una artesana del lenguaje difícil de captar. En la introducción de "The Place of Miracles", ella misma define su lengua como "my alchemist's bag, usually open", ${ }^{6}$ lo que da fe del grado - y dificultad - de su experimentación con las palabras.

La autora ha reconocido en alguna ocasión su afinidad con Patrick Kavanagh porque no en vano ambos proceden de Monaghan y han compartido paisaje y paisanaje: "me encantaba pensar que Kavanagh procedía de aquellos lugares y que en un tiempo había visto todo lo que yo ahora contemplaba". 7 Y como él, demuestra poseer un extraordinario sentido de lo inmediato, del momento presente - hic et nunc - de la solemnidad de lo que acontece ante nuestros ojos y que en sí mismo posee la máxima categoría artística. Para ella, como para Kavanagh, el poeta no es un mero observador o transcriptor sino parte irrenunciable del mundo que describe y que sacraliza en el acto de nombrarlo poéticamente.

De cuanto precede se deriva mi responsabilidad como traductora, algo que asumo con no poco temor y con suma modestia. No cabe duda que esta experiencia me ha ensanchado la mente y el espíritu y que me he beneficiado de la capacidad regeneradora de la traducción que la propia autora glosa en su poema "Growing into Irish through Galicia". Pese a todo, yo sólo aspiro, emulando uno de los versos de "Hungary", a que estas traducciones hagan de Mary O'Donnell una nativa para aquellos lectores hispanoparlantes que no tienen la fortuna de leerla y disfrutarla en su versión original. Que así sea.

6. " mi bolsa de alquimista, normalmente abierta" . O'Donnell, Mary, "Introduction" The Place of Miracles. New and Selected Poems. Dublin: New Island Books, 2006.

7. González Arias, Luz Mar .Ob. Cit. 2008: 147. 


\section{Poems}

(Used by kind permission of the author)

Feeding the Crone ${ }^{1}$

Hungary ${ }^{1}$

The Cosmos Ticked Silently ${ }^{3}$

Spring Funeral ${ }^{4}$

Eden $^{5}$

Buzzard $^{6}$

Pleasure $^{7}$
Alimentando a la vieja bruja

Hungría

El cosmos latía en silencio

Funeral de primavera

Edén

Águila ratonera

Placer

1. Publicado en la revista literaria The Stony Thursday Book en 2012

2. Publicado en The Warwick Review en 2012

3. Publicado en The Irish Times, 22 de diciembre del 2013

4. Inédito

5. Publicado en la revista literaria The Stony Thursday Book en 2012

6. Inédito

7. Inédito

\section{Feeding the Crone}

The crone is knocking on my door.

Despite myself I open.

A north wind gusts in.

'Any chance of a cup of tea?' she asks, conciliatory,

(as if we hadn't been through this rigmarole several times already).

I wouldn't grudge a person the chance to wet their lips,

I'd throw in a biscuit or two, not to mention a slice of the cake my daughter made yesterday. That's what she needs! A young girl's cake, free-range eggs, flour, country butter, rich with youth.

I slip the crone a huge triangle, knowing she won't say no.

Blue and white frosting sidle down the sides, stick to her fingers, make their way to sweeten her mouth.

She swallows and licks, casts a glance at the cake a second time.

Again, I cut deep, to fill her sagging belly. What else would I do?

By the time she's done, she hesitates in the doorway, a smile almost cracking her jowls.

'That was some cake,' she whispers, turning away, patting herself as if she was pregnant.

\section{Alimentando a la vieja bruja}

La vieja bruja llama a mi puerta. A mi pesar le abro.

Un viento norte entra a rachas. “¿Nos tomamos un té?" pregunta, conciliadora,

(como si no hubiéramos oído este cuento varias veces ya).

Yo no escatimo a nadie una ocasión para mojar sus labios, más una o dos galletas, no digamos un trozo del pastel que hizo ayer mi hija. ¡Eso es

lo que ella necesita! Un pastel de chica joven, huevos de granja, harina, mantequilla de pueblo, rico en juventud.

Le paso a la bruja un triángulo enorme, sabiendo que no dirá que no.

El glaseado azul y blanco se escurre por los bordes, se le pega a los dedos, se abre camino para endulzar su boca. Ella traga y lame, mira el pastel de nuevo.

Le corto más, para llenar su vientre caído ¿Qué más podría hacer?

Ella, en cuanto termina, vacila en el umbral,

una sonrisa casi agrieta sus carrillos.

"Eso sí es un pastel", susurra al alejarse, pasándose la mano como si estuviera embarazada. 


\section{Hungary}

came to me in stamps.

"Magyar Posta" ice-skaters, delicate as Empire porcelain, a fish, an astronaut and rocket, a silvery boy on 1960s skis. I understood only difference.

Now, flying home from Budapest, I touch the pages of my poems, freshly minted in translation. Now I really don't get them, but did I ever? The words will make me briefly native to a coffee-slugging morning reader on the Vaci Ut, who may not understand, even in his own tongue.

The lines shimmer as night slips through the tilting crowded cabin. Again I press fingers to page, blind, as if by touch I could capture a fish, an astronaut, a rocket, or those elegant, ice-cutting skaters.

Outside, clouds I cannot see

busily translate country to country.

\author{
Hungría \\ vino a mí en sellos de correos. \\ "Magyar Posta" patinadores sobre hielo, delicados \\ cual porcelana fina, un pez, un astronauta \\ y un cohete, un chico plateado sobre unos esquís de los 60 . \\ Solo entendí la diferencia. \\ Ahora, volando a casa desde Budapest, \\ toco las páginas de mis poemas, recién acuñados \\ en una traducción. La verdad es que no los entiendo, \\ pero, ¿alguna vez sí? Las palabras me harán \\ nativa por un rato para un lector que toma \\ de mañana un café en Vaci Ut, y que quizá no entienda, \\ ni siquiera en su lengua. \\ Los versos relucen mientras la noche escapa \\ por la repleta cabina inclinada. Otra vez \\ mi dedo presiona la página, a ciegas, como si al tacto \\ pudiera capturar un pez, un astronauta, un cohete, \\ o los patinadores elegantes sobre el hielo. \\ Fuera, unas nubes que no puedo ver \\ se mueven afanosas de país a país
}

\section{The Cosmos Ticked Silently}

They came with fragments hacked from arid inland ravines, golden brown or flecked like a pink moon

before a storm. They crammed it before the journey, in a casket. Naturally, other gifts - gold, myrrh, some balming oils for the mother, the spent sack of her belly - were brought to the narrow refuge where the new family huddled

beside asses, some ailing sheep, one wild dog drawn to the smell of birth blood. Steam from bestial

droppings rose in the night. But the al-luban from Oman was fragrant. Even before it burned, the young pair,

exhausted after the journey, stirred, felt purposeful again, the future grisling and gurning as the mother marvelled at the child's nudging lips, his tiny fists that opened and closed like desert anemonae. Overhead, a bright, steering cosmos ticked silently, the enveloping dark pricked with light years of stars, indifferent to it all.

\section{El cosmos latía en silencio}

Vinieron con troncos talados de áridos barrancos tierra adentro, de dorado marrón o moteados como una luna rosa antes de una tormenta. Lo embutieron todo en un cofre antes del viaje. Por supuesto, otros regalos - oro, mirra, aceites balsámicos para la madre, el saco vacío de su vientre - fueron llevados al estrecho refugio donde la nueva familia se acurrucó 
al lado de los asnos, de unas ovejas achacosas, de un perro salvaje atraído al olor de la sangre del parto. En la noche ascendía el vapor de excrementos animales. Pero el al-luban de Omán tenía fragancia. Antes que se apagara, la joven pareja, rota tras el viaje, se sintió de nuevo despierta y resuelta, el futuro cambiando y torciéndose mientras la madre se maravillaba de los labios del niño rozándose, de sus puñitos abriendo y cerrándose como anémonas del desierto. Guiando en lo alto, un cosmos brillante latía en silencio, envuelto en la noche pinchada por años luz de estrellas, indiferente a todo.

\section{Spring Funeral}

We move as actors in the family opera, sing our best and worst roles. Sparrows nest in the belfry,

lilac trembles beneath gusted cloud. Grief plays some part, and other things not intended for this day.

There's Lear, raging at the loss of all held dear, while Fool son, despised, points again to the error of his ways.

There's Daughters Elder and Younger, united as they move across the nave towards Cousin, prefecting and bright-eyed, never more alive as she consoles. Also Unwanted Uncle whose crime is never clear,

Maiden Aunt who remembers the crime though also his birthday. There too, Middle Child, a trembling veil over her eyes,

the skewer of her unhappiness in a tight percussion of heel-beat, as she fills the church from door to pew. How well played the roles,

our every hour a rehearsal for something guessed at, feared, yet never known by heart. We crowd close

around a gaping grave no fake green grass can temper.

The whole earth sings a clattering aria

as clods crumble on coffin lid, repeat the lines

to the beat of our hearts: one day, understudy, the next,

the curtain call we always knew would come.

\section{Funeral de primavera}

Nos movemos como actores en la opera familiar, cantamos nuestros mejores y peores papeles. Los gorriones anidan en el campanario, tiembla la lila bajo rachas de nube. El dolor juega algún papel, y otras cosas no destinadas a este día.

Está Lear, furioso por perder todo lo que quería, mientras el Bufón hijo, despreciado, le acusa otra vez del error de sus actos.

Están las Hijas Mayor y Menor, unidas mientras cruzan la nave hacia la Prima, celadora 
de ojos brillantes, nunca tan viva como cuando consuela.

También el Tío Non Grato, cuyo crimen está por aclarar,

La Tía Soltera que recuerda tanto su crimen como su cumpleaños.

Y la Hija Mediana, con un velo temblándole en los ojos,

Y la brocheta de su infelicidad en una tensa percusión de tacones, mientras llena la iglesia desde la puerta al banco. Qué papeles tan bien interpretados,

cada hora nuestra es un ensayo de algo adivinado, temido, pero nunca aprendido de memoria. Nos apiñamos

alrededor de una tumba abierta que un falso césped no logra atenuar.

La tierra entera canta un aria estruendosa

mientras los terrones se desmigan sobre el féretro, repiten los versos

con el latido de nuestros corazones: un día, el suplente, el próximo,

la llamada a escena que siempre supimos que vendría.

\section{Eden}

Someone long ago had planted Beauty of Bath

in the Protestant orchard of my childhood.

My mother's orchard. Wasps

devoured that neglected sweetness

she never saw as she drove out,

forgetful of us as we lounged in the crotches

of old trees, emperors, home from war.

To get there was to do battle with nettles, buzzing insects, cock-chafers and frogs, our own myths of serpents. We were troops on the march, Hannibal crossing the Alps with dogs for elephants. Sometimes fear set us back, until someone became an Alexander, play-acting courage to keep the troops in the mood.

\section{Ahead}

lay an India - apple-trees, the potioned poms, flesh creamy and flush-veined. All we wanted, ruby-fleshed lanterns: such maps of the senses, an antidotal release on our tongues, that beautiful sting .

\section{Edén}

Alguien había plantado tiempo atrás manzanas de Bath en el huerto Protestante de mi infancia.

El huerto de mi madre. Las avispas devoraron aquel dulzor abandonado que ella nunca veía cuando se marchaba, sin ocuparse de nosotros que ganduleábamos en las orquillas de árboles viejos, emperadores, de vuelta a casa tras la guerra. 
Llegar allí era batallar con ortigas, con zumbido de insectos, abejorros y ranas, nuestros propios mitos de serpientes. Éramos tropas en marcha, Aníbal cruzando los Alpes con perros en lugar de elefantes. A veces el temor nos paraba, hasta que alguien hacía de Alejandro, simulando valor para mantener a las tropas con ánimo.

Ante nosotros

se extendía una India - manzanos, la pócima de las pomas de piel cremosa y venas encendidas.

Todo cuanto queríamos, linternas con piel rubí: esos mapas de los sentidos, un estallido de antídoto en las lenguas,

aquel bello escozor.

\section{Buzzard}

Came August, it furrowed beneath the sun, above the big field, low enough to sight the scallop of wing-tip, that opaque span widening as it steadied in a downwards sweep. Sometimes it sat on electric cables that festooned and crackled across the sky, shouldered in on itself briefly, a pause to fall to flight, resume that longing cry.

It cut like scimitars beneath cloud-trails, sky-pirate to the stolen, golden days. And we in the garden, never too lost in sunbathing, or unrubbling new potatoes from the giving ground, looked up, and up at a world still tilting wildness from its cup.

\section{Pleasure}

Slack and easy as dropped sails, the rook's wings: on the high bank beneath the trees, sun melted deeply into the stiff mail of every feather. Warmth penetrated to flesh. The black head arched, shivered, eyes attentive to the sky, dark sails of wing silky beneath shoulders, on this noon-lit grassy slope, freed to rest, this small creature - less than a molecule

in the outspread dozing of a cosmos freighted with the care of every creature sat softly pleasured on grass, yet frail too despite mail of feather and stone-hard beak, in the respite of heat, the wings at rest, shuddering, first summer out of the nest.

\section{Águila ratonera}

Vino en Agosto, hacía surcos bajo el sol sobre el extenso campo, a baja altura para ver el festón de la punta del ala, ese palmo opaco que se abría mientras planeaba y bajaba en un arco. A veces se posaba en los cables eléctricos que adornaban el cielo crepitando, se arrebujaba un rato, una pausa para empezar el vuelo, para reanudar aquel grito anhelante.

Cruzaba como cimitarras bajo estelas de nubes, pirata del cielo en los días robados, dorados. Y en el jardín nosotros, nunca del todo absortos tomando el sol o desenterrando patatas nuevas del dadivoso suelo, mirábamos arriba y arriba, hacia un mundo que aún vertía lo salvaje de su copa.

\section{Placer}

Sueltas y quietas como velas plegadas, las alas del grajo: bajo los árboles de la alta orilla el sol derritiéndose hasta el fondo de la dura malla de las plumas. El calor penetraba en la carne. La cabeza negra se arqueaba, temblaba, los ojos fijos en el cielo, en los costados oscuras velas de sedosas alas, Sobre este césped con luz de mediodía, libre para el descanso, esta criaturita - menos que una molécula

en el extenso dormitar de un cosmos fletado para cuidar a cada criatura se posó en la hierba complacida y sin ruido, aunque frágil a pesar de la malla de pluma y del pétreo pico, con el calor en tregua, las alas en reposo, estremecida, primer verano fuera del nido. 